DOI: $10.25100 /$ pfilosofica.v0i53.11533

\title{
MÁS ALLÁ DEL PUEBLO Y DE LA CLASE OBRERA INDUSTRIAL. LA TEORÍA POLÍTICA DE LA MULTITUD DE PAOLO VIRNO
}

\{ Andrea Fagioli

CONICET - Universidad Nacional de San Martín, Argentina

\begin{abstract}
Resumen
Paolo Virno es uno de los filósofos que han dedicado más esfuerzos a la reflexión sobre la multitud, considerado un concepto adecuado para pensar la subjetividad política a la altura de los tiempos presentes. Virno es un pensador eminentemente político, sus reflexiones siempre echan raices en inquietudes político-militantes; sin embargo, muchas veces la dimensión politica de su pensamiento se encuentra en la reflexión sobre los temas eternos. Reconstruir una teoría política de la multitud en Virno implica por lo tanto componer un collage que vaya más allá de su mero cuerpo a cuerpo con la tradición filosófico-política, y en el cual emerjan los elementos que caracterizan la multitud, su politicidad intrínseca y, paralelamente, las bases para una política alternativa.
\end{abstract}

Palabras clave: multitud; teoría politica; postfordismo; subjetividad; pueblo.

Cómo citar este artículo: Más allá del pueblo y de la clase obrera industrial. La teoría política de la multitud de Paolo Virno. Praxis Filosófica, (53), 205-224. https://doi.org/10.25100/pfilosofica. v0i53.11533

Recibido: 9 de marzo de 2021. Aprobado: 8 de junio de 2021. 


\title{
Beyond the People and the Industrial Working Class. Paolo Virno's Political Theory of the Multitude
}

\author{
Andrea Fagioli ${ }^{1}$
}

\begin{abstract}
Paolo Virno is one of the philosophers who have dedicated more attention to the reflection on the multitude, considered an adequate concept to think the political subjectivity at the level of the present times. Virno is an eminently political thinker, his reflections are always rooted in political-militant concerns, however, many times the political dimension of his thought is found in the reflection on the eternal issues of the philosophy. Reconstructing a political theory of the multitude in Virno, therefore, implies composing a collage that goes beyond its mere hand-to-hand with the philosophicalpolitical tradition and in which emerge the elements that characterize the multitude, its intrinsic politicization and, in parallel, the basis for an alternative politics.
\end{abstract}

Keywords: Multitude; Political theory; Postfordismo; Subjectivity; People.

\footnotetext{
${ }^{1}$ Licenciado en Filosofía por la Universitá de Perugia, Italia; Magister en Periodismo por la Universitá de Sassari, Italia; y Doctor en Filosofía por la Universidad Nacional de San Martín-UNSAM. Actualmente se desempeña como tutor del seminario de tesis en la Maestría en Desarrollo Humano de Flacso-Argentina y es becario posdoctoral de CONICET, con un proyecto sobre capitalismo de plataformas que lleva adelante en la Escuela IDAES (UNSAM). Sus principales intereses de investigación son el capitalismo contemporáneo, los movimientos sociales, el problema de la clase y el debate sobre neoliberalismo.
}

ORCID: 0000-0002-0252-8920 E-mail: andrea.fagioli81@gmail.com 


\title{
MÁS ALLÁ DEL PUEBLO Y DE LA CLASE OBRERA INDUSTRIAL. LA TEORÍA POLÍTICA DE LA MULTITUD DE PAOLO VIRNO
}

\author{
Andrea Fagioli
}

I.

El filósofo italiano Paolo Virno es un pensador militante atípico. Su filosofía no puede ser comprendida cabalmente sin tener en debida cuenta el hecho que está movilizada por sus inquietudes políticas ${ }^{2}$, pero a la vez su pensamiento político y su militancia pasan muy a menudo por la reflexión sobre los "temas eternos". Es obvio, por lo tanto, que cuando hablamos de política en Virno no nos referimos, o por lo menos no solamente, a aquella que tiene su "sede" en el Estado y al pueblo como sujeto. Reflexionando en y sobre una coyuntura marcada, por un lado, por la reconfiguración a nivel global del Estado y, por otra parte, por una transformación del modelo productivo, lo que le interesa a Virno -así como a toda la tradición postoperaista, de la cual es uno de los teóricos más importantes- es el sujeto político que se constituye en el punto donde se encuentran la crisis de la forma Estado y las transformaciones del capitalismo (Chignola y Mezzadra, 2012).

Para pensar una subjetividad a la altura de los tiempos presentes, Virno retoma de los albores de la filosofía política moderna el concepto de multitud $^{3}$. Para el filósofo, si en el siglo XVII la multitud fue expulsada del horizonte hegemónico del pensamiento político moderno -que ha puesto en su centro a su contrincante, el concepto hobbesiano de pueblo-del cual

\footnotetext{
${ }^{2}$ Sobre esta cuestión me permito remitir a mi Fagioli, 2019.

${ }^{3}$ Virno no es el único, piénsese en los trabajo extremadamente influyentes de Michael Hardt y Antonio Negri $(2002 ; 2004)$
} 
pasó a constituir el límite negativo ${ }^{4}$, en el momento en que el horizonte político moderno está frente a una crisis, en el marco de la cual "las viejas categorías explotan y es preciso acuñar otras nuevas" (Virno, 2003, p. 15), la multitud exhibe una sorprendente vitalidad.

Sin embargo, en un momento histórico en que es cada vez más difícil separar lo político de lo económico-pruductivo y de lo cultural, la multitud no se coloca solo más allá del pueblo, sino también más allá del otro grande sujeto colectivo que ha habitado la época moderna: la clase obrera industrial. Pensar una teoría política por venir o una teoría política del éxodo -el filósofo usa ambas fórmulas para pensar una teoría para la multitud-, significa elaborar herramientas conceptuales para un sujeto que pueda aspirar paralelamente a una liberación política y económica, es decir liberación de la soberanía (no solo estatal) y liberación de la explotación capitalista.

En los textos de Virno no se encuentran recetas para la acción política, y tampoco análisis sobre la globalización o el papel del Estado -como sí las hay en otros autores de la misma perspectiva política-, la reflexión del filósofo apunta siempre a las condiciones de posibilidad, evidenciando las bases para posibles alternativas. Por esta razón, este artículo no tiene un desarrollo lineal, sino que los apartados que lo componen se implican mutuamente y trata de componer un collage de piezas necesarias para entender los elementos de la teoría política de la multitud que propone Virno. En las páginas que siguen voy a reconstruir la manera en que Virno piensa los modos de ser que caracterizan a la multitud (II); posteriormente me voy a centrar en la manera en que el filósofo considera el concepto de multitud adecuado para dar cuenta de la etapa contemporánea del capitalismo (III); sucesivamente voy a intentar dar cuenta de los elementos de la antropología política que subyacen a todas las formulaciones de Virno (IV); y finalmente voy a delinear el cuerpo a cuerpo de Virno con los propios fundamentos de la filosofía política moderna (V).

\section{II.}

En las primeras páginas de Gramática de la multitud, Virno (2003) nos propone una definición mínima del concepto, que es importante tener en cuenta. Ahí la multitud es descrita en términos de una

\footnotetext{
${ }^{4}$ En palabras de Hobbes (2007), quien abre una senda el pensamiento político en la cual se inscribe la filosofía política moderna hegemónica, "una multitud de hombre se hace una persona cuando son representados por un hombre o una persona siempre (...) pues es la unidad del mandatario, no la unidad de los representados, lo que hace de él una persona una" (p 158). En este sentido la multitud constituye lo prepolítico, si se piensa en términos cronológicos, y también el límite más allá del cual la política no puede existir.
} 
pluralidad que persiste como tal en la escena pública, en la acción colectiva, en lo que respecta a los quehaceres comunes (comunitarios) sin converger en el Uno (...) Multitud es la forma de existencia social y política de los muchos en tanto muchos (pp. 11-12).

En ese pasaje, Virno subraya la exterioridad de la multitud con respecto a lo que podemos definir el dispositivo conceptual de la modernidad políticas, es decir la producción de los sujetos colectivos por medio del pacto y la representación (véase al respecto Duso, 2015). Pero dejemos para más adelante estas reflexiones y veamos las tres aproximaciones a la multitud que propone el autor.

En el parágrafo 40 de El ser y el tiempo, Martin Heidegger (2009) distingue miedo y angustia; el primero hace referencia a un hecho específico, mientras que la segunda no tiene una causa identificable y es provocada por la simple exposición al mundo. Virno (2003) traslada esta diferenciación al terreno filosófico-político, ahí el miedo es asociado al concepto de pueblo, basado en una "neta separación entre un 'adentro' habitual y un 'afuera' ignoto y hostil" (p. 21) . En este sentido, "los peligros filtrados y atenuados por el ethos comunitario provocan solamente miedo: de ellos hacemos una razón, se los puede prever y contener, cada tanto expulsar" (Virno, 2017, p. 79). Si bien acá Virno parece pensar más en una sociedad que -parafraseo a Foucault- "tiene que ser defendida", el mismo ingreso a la "vida política", tal y como ha sido pensado en la modernidad, puede ser considerado como el pasaje de un estado (de naturaleza) en el cual la vida estaba incondicionalmente expuesta a una difusa amenaza de muerte, a un estado (civil) en que esta está expuesta a una amenaza que se encuentra únicamente en manos del soberano.

Sin embargo, frente al derrumbe de las comunidades sustanciales, la separación entre interno y externo, y por lo tanto entre miedo y angustia, se disuelve y la coincidencia de los dos pasa a caracterizar la condición común contemporánea, que es un elemento fundamental para el concepto mismo de multitud. En este sentido "los 'muchos' son efectivamente tales en cuanto comparten la experiencia del 'no sentirse en la propia casa"' (Virno, 2017, p. 81). Dicho en otras palabras, en el contexto que se suele llamar globalización, en el marco del cual las comunidades nacionales muestran más de una

\footnotetext{
${ }^{5}$ Vale la pena recordar que, para Hobbes (2009), "el pueblo es una sola unidad, que tiene una sola voluntad (...) Nada de esto se puede decir de una multitud” (p. 158).

${ }^{6}$ Roberto Esposito (2003) diría, con un vocabulario un poco diferente, que "el Estado no tiene el deber de eliminar el miedo, sino de hacerlo "seguro"' (p. 61).
} 
grieta $^{7}$, emerge en primer plano una subjetividad colectiva compuesta por singularidades que están acostumbradas a no tener hábitos y cuya relación con el mundo no es atenuada o velada por aquellas comunidades.

Sin embargo - llegamos así a la segunda aproximación a los muchosla tesis de Virno es que la multitud no rechaza la unidad que caracteriza el pueblo, sino que la redetermina. Ya no hay Uno en el sentido de una promesa, el Estado hacia el cual convergen los individuos, sino como premisa constituida por las facultades comunes que los seres humanos comparten. Entonces, en el momento en que las comunidades sustanciales no pueden ofrecer reparo a la superposición de miedo y angustia, los recursos esenciales para protegerse de la peligrosidad del mundo son identificados, por el filósofo, en el concepto aristotélico de lugares comunes (topoi koinoi), un concepto lingüístico con el que el Aristóteles (1971) indica las formas lógicas y lingüísticas de valor general, que Virno (2003) llama "la estructura ósea de cada uno de nuestros discursos" (p. 26).

En tanto tales, en la Retórica estos lugares comunes son pensados como una trama invisible de la vida de la mente $^{8}$, pero en la lectura de Virno la peculiaridad de la época contemporánea es que -lo veremos en el próximo apartado- esta vida de la mente se ha tornado el principal recurso productivo del capitalismo postfordista, donde adquiere inmediata visibilidad lo que Marx llama general intellect, pensamiento en tanto recurso público, transindividual 9 .

De esta manera, la unidad de la multitud es distinta de la del pueblo, porque en el segundo caso "el Uno es el punto final de un movimiento centrípeto. La multitud, en cambio, es el punto final de un movimiento centrífugo; del Uno a los Muchos"(Virno, 2003, p. 35). Y esta unidad que la multitud tiene a sus espaldas es, justamente, la de las aptitudes lingüísticas y comunicativas de la especie, que llegan a ocupar el primer plano no por

${ }^{7}$ Virno, quien volvió a publicar en italiano La idea de mundo en 2015 (el original es de 1994), es perfectamente consciente del rebrote nacionalista que atraviesa el continente europeo y no solo (piénsese por un lado en Salvini, Le Pen, Orban, Johnson, etc. y, por otro lado, en Trump y Bolsonaro). La publicación de ese texto tal cual estaba, parece más bien demostrar la voluntad de reafirmar su hipótesis. En ese sentido, los nuevos nacionalismos representarían el sueño imposible de volver a un miedo seguro y controlado y serían justamente una respuesta a aquella condición de no sentirse en casa propia.

${ }^{8}$ Los que se hacen visibles son los que Aristóteles (1971) llama topoi idioi, los lugares especiales, modos de decir que pertenecen exclusivamente a ámbitos específicos de la vida social.

${ }^{9}$ Virno extende el concepto marxiano de general intellect y no se refiere solo a la fuerza objetivada de conocimiento" (Marx, 1989, p. 230), es decir saber cristalizado en las máquinas, sino a las aptitudes genéricas y transindividuales de los seres humanos, que se tornan un elemento clave de la producción postfordista. 
una decisión soberana, sino por necesidad, porque "constituyen una forma de protección en una sociedad privada de comunidades sustanciales (o sea, de 'lugares especiales')" (Virno, 2003, pp. 35-36).

Sin embargo -tercera y última aproximación- la publicidad del intelecto es ambivalente, como ambivalente, en el título de una compilación de textos muy importantes de Virno ${ }^{10}$, es la multitud. Por un lado, el general intellect es, como hemos visto, la base de una producción social que va más allá del trabajo asalariado y que "desarrolla la técnica más allá de la máquina" (Fujita Hirose, 2006, p. 35). Pero también hay una segunda dimensión, más inmediatamente política, del general intellect-en cuyo nombre resuena una alusión polémica a la Volonté Générale de Jean-Jacques Rousseau-, donde reside la ambivalencia. El general intellect puede ser, para Virno, "la base de instituciones políticas que dejan atrás al Estado, sus aparatos administrativos centralizados, la obligación de obedecer" (Fujita Hirose, 2006, p. 35), es decir que puede dar lugar a una esfera pública no estatal, en la cual los muchos puedan ocuparse en tanto muchos de los asuntos comunes. Sin embargo, si el carácter público del intelecto puede ser la base de una teoría política por venir, al mismo tiempo "allí donde no se articula en una esfera pública, se traduce en una proliferación incontrolada de jerarquías, tan infundadas como resistentes" (Virno, 2003, p. 34).

\section{III.}

En este segundo apartado me voy a enfocar en el hecho de que, como ya emergió, la multitud no se opone para Virno al concepto de clase obrera, sino que va más allá de una forma históricamente determinada de aquella, la clase obrera industrial. En palabras del filósofo:

la clase obrera no coincide, ni en Marx ni en la opinión de cualquier persona seria, con ciertos hábitos, ciertos usos y costumbres, etc. "Clase obrera" es un concepto teórico, no una foto recuerdo: remite al sujeto que produce plusvalor absoluto o relativo. Y bien, la clase obrera contemporánea, el trabajo vivo subordinado, su cooperación cognitivo-lingüística, tiene los rasgos de la multitud, antes que los del pueblo (Virno, 2003, p. 39).

${ }^{10}$ Me refiero a Ambivalencia de la multitud (Virno, 2011b), compilación que presentó a lectores hispanohablantes textos fundamentales del filósofo, entre los cuales dos artículos sobre los cuales voy a volver en repetidas ocasiones más abajo: "El llamado 'mal' y la crítica del estado" y "Chiste y acción innovadora". 
Es de fundamental importancia entender, a partir de lo que vimos en el apartado anterior, qué significa que la clase obrera tenga hoy los rasgos de multitud y los efectos que esta lectura conlleva.

Vayamos por partes. Refiriéndose al operaismo italiano, Marco Mazzeo (2015) ha afirmado que "las investigaciones de esta filosofía radical pueden ser leídas como un comentario continuo a un único pasaje aristotélico" (p. 181), es decir que se insertan en el campo delimitado por las dos definiciones que Aristóteles da del ser humano: "animal con lenguaje" y "animal político". En este sentido, sostiene el mismo Mazzeo (2015), por un lado se comprende "la politicidad de los sapiens solo si se toma en cuenta su lingüisticidad; viceversa es posible asir hasta el final el significado de la lingüisticidad de los sapiens sólo si se toma en cuenta su carácter político" (p. 181).

Dejando de lado si es correcto atribuir esta peculiaridad a la historia entera del operaismo, lo que es importante aquí destacar es que desde esa perspectiva, la plena sinonimía de las dos definiciones, lejos de subordinar la segunda ("animal político") a la primera ("animal con lenguaje"), enfocándose en los usos políticos de la palabras, plantea "la politicidad intrínseca del lenguaje" (Virno, 2005, p. 58). Esto es un elemento a tener sumamente en cuenta.

Si nos trasladamos al terreno de la producción, vemos que autores fundamentales de la tradición marxista, desde el propio Marx hasta Lenin y Lukacs, así como filósofos contemporáneos-emblemática es la distinción habermasiana entre acción comunicativa y acción instrumental (Habermas, 1989)-, han retomado "a piene mani" (Virno, 2011a) una concepción teleológica del trabajo que, filtrada por la lectura de Hegel ${ }^{11}$, coloca el lenguaje por fuera de la producción. Semejante concepción, que podía tener cierta utilidad en el marco de la producción industrial, vendría a mostrar todos sus límites en el momento en que la comunicación entra en el espacio productivo.

Me parece fecundo, al respecto, retomar algunos análisis de autores que comparten con Virno la misma perspectiva y experiencia políticas -en un sentido amplio- para mostrar de manera muy gráfica ese cambio de paradigma y aclarar así su punto de vista ${ }^{12}$. En la novela símbolo de la

${ }^{11}$ En las clases dictadas en Jena en 1803-1804, Hegel (2006) había estudiado el problema del trabajo desde dos distintos puntos de vista: en primer lugar como relación finalista aislada entre el individuo y el objeto de su necesidad particular, mediada por la herramienta de trabajo. En segundo lugar como actividad que, aun manteniendo invariada su estructura originaria (relación entre individuo y objeto de su necesidad mediada por una herramienta de trabajo) es sobredeterminada desde afuera y retrospectivamente por relaciones distributivas, jurídicas e institucionales.

${ }^{12}$ Abordé estas cuestiones de manera más sistemática en mi Fagioli, 2016a. 
temporada del obrero-masa ${ }^{13}$, Lo queremos todo de Nanni Balestrini (2006), el protagonista era un tipo cualquiera que pasaba, al igual que sus colegas cualquiera, de una tarea a otra a lo largo de la cadena de montaje y que saltaba de un sector de la industria a otro, completamente distinto, después de unos pocos días de-diríamos hoy-training. Alfonso, el trabajador que Balestrini retrata con enorme fuerza narrativa, era retribuido por gastar genéricamente energía en una máquina, alrededor de la cual hacía movimientos que, desde el punto de vista físico, eran extremadamente variados y entraban en la producción de valores de uso completamente distintos: sanitarios, dulces, automóviles, etc. Se trata de movimientos heterodirigidos, ya que, como sostiene Christian Marazzi (2003) en un texto muy influenciado por el trabajo conceptual de Virno, "la cadena de montaje era muda porque ejecutaba mecánicamente las instrucciones elaboradas en las oficinas de los trabajadores de cuello blanco (white collar)" (p. 14).

Algunas décadas después, centrándose en el análisis de lo que llama semiocapitalismo o capitalismo semiótico, Franco "Bifo" Berardi subrayaba que los trabajadores cognitivos, al contrario, hacen físicamente los mismos movimientos: están sentados frente a la computadora, tipeando, la mayoría del tiempo. Sin embargo, sus trabajos son incomparables, ya que requieren competencias específicas, manejo de lenguajes y códigos que no tienen nada que ver uno con otro. Si mientras más el trabajo industrial se simplifica más es intercambiable, "frente a la computadora, y conectados a la máquina universal de elaboración de comunicación, los terminales humanos ejecutan todos los mismos movimientos físicos" (Berardi, 2009, p. 98). Es decir que, a una mayor simplificación del trabajo desde el punto de vista físico, corresponde una menor intercambiabilidad de conocimientos, capacidades y prestaciones, que se tornan más complejos.

Sin embargo, si Bifo parece pensar en una minoría de trabajadores del terciario avanzado, altamente especializados, para Virno en el capitalismo contemporáneo lo que es puesto a trabajar es general intellect. En el postfordismo, es la misma cadena de producción que se hace hablante, comunicante (Marazzi, 2003) y esto es evidente, para Virno, si se consideran los principales requisitos que se les exigen hoy a los trabajadores, a quienes se le pide que estén

habituados a moverse de un lado a otro, que sean capaces de acomodarse a las más bruscas reconversiones, que sean adaptables para cambiar de una dependencia a otra, que sean dúctiles para cambiar las reglas del juego, que

\footnotetext{
${ }^{13}$ Concepto acuñado por los autores operaistas para indicar a los obreros de las fábricas fordistas, véase al respecto Negri, 1980.
} 
sepan llevar adelante interacciones lingüísticas banales; que demuestren destreza para elegir y sepan manejar diversas alternativas. Pues bien: estos requisitos no son fruto del disciplinamiento industrial, sino sobre todo el resultado de una socialización que tiene su centro de gravedad fuera del trabajo (...) La empresa postfordista usufructúa de este hábito de no tener hábitos, este adiestramiento en la precariedad y la variabilidad (Virno, 2003, p. 91).

Dicho en otras palabras, el trabajor postfordista no tiene que incorporar y poner en acto unos actos potenciales ${ }^{14}$, sino que tiene que recurrir a la indeterminación propia del animal lingüístico, ser inespecializado, su politicidad.

Ahora bien, al nivel del trabajo el postfordismo se caracteriza, según Virno, por la crisis de la experiencia humana tal y como había sido sistematizada por Aristóteles (2007), quien distinguía poiesis, que remite al intercambio orgánico con la naturaleza; praxis que se refiere al ámbito de las relaciones sociales; y biós theoreticos que tiene que ver con la meditación del pensador solitario.

214 Se trata de una división que no solo ha sido retomada en el debate filosófico, sino que de alguna manera ha sido asumida por el sentido común. De hecho, Virno (2003) recuerda que cuando se inició en la militancia política, en la Italia fordista de los años 60 , esa tripartición le parecía "tan irrefutable como una percepción táctil o visual” (p. 47). Es decir que "no era necesario haber leído la Ética Nicomaquea de Aristóteles para saber que trabajo, acción política y reflexión intelectual constituían tres esferas regidas por criterios heterogéneos" (Virno, 2003, p. 42).

Haciendo lo que él mismo define un "uso crítico de los adversarios" (Milazzo, 2011), Virno reconoce a Hannah Arendt (2009) el mérito de haber comprendido antes que nadie, que las fronteras entre trabajo y política se habían hecho borrosas en el siglo XX. Sin embargo, si para la filósofa alemana era la política la que imitaba el trabajo y que había "fabricado" objetos nuevos como el Estado, el partido o la Historia, para Virno las cosas se habían dado exactamente al contrario.

Entonces, si, como sostiene Gramsci $(1981)^{15}$, en el fordismo el cerebro permanecía afuera de la producción y solo al salir de la fábrica el trabajador

\footnotetext{
${ }^{14}$ Virno lee de manera muy peculiar el concepto aristotélico de potencia, introduciendo entre potencia y acto un tercer elemento, los actos potenciales. En este sentido, la técnica coincidiría con la incorporación de actos potenciales, mientras que el trabajo postfordista estribaría directamente en la potencia. Por razones de espacio no me puedo extender aquí sobre esta cuestión y remito a mi Fagioli, 2020.

${ }^{15} \mathrm{Me}$ refiero, en particular, a "Americanismo y fordismo", "Cuaderno 22", tomo 6.
} 
leía el diario, militaba, pensaba, dialogaba, etc., en la etapa postfordista el lenguaje está incluído en el tiempo-espacio de la producción. Si se parte del presupuesto de la politicidad del lenguaje, en el trabajo contemporáneo la politicidad intrínseca del hombre es llevada dentro de la "oculta sede de la producción" (Marx, 2010, p. 214).

\section{IV.}

Virno ha expresado en varias entrevistas (Colectivo Situaciones, 2011; Rispoli, 2016) que el terreno de la antropología constituye un campo de batalla particularmente importante en términos políticos. La naturaleza humana, desde su punto de vista, "nunca dispone una solución: es siempre parte del problema (Colectivo Situaciones, 2011, p. 9); por esto, y en polémica con las posiciones de Noam Chomsky, Virno considera el hecho de deducir una estrategia política a partir de los rasgos distintivos de nuestra especie la "más tonta de las tonterías" (Colectivo Situaciones, 2011, p. 8) que se pueden decir al indagar la relación entre naturaleza humana y lucha política.

En su lectura, teorías de las instituciones políticas y concepción de la naturaleza humana se implican mutuamente, al punto que

no hay indagación imparcial acerca de la naturaleza humana que no lleve consigo, como un pasajero clandestino, al menos un esbozo de una teoría de las instituciones políticas (...) Y viceversa: no hay teoría de las instituciones políticas digna de este nombre que no adopte, como su oculto presupuesto, una u otra representación de los rasgos que distinguen al Homo sapiens de las otras especies animales (Virno, 2011b, p. 121).

Sobre este terreno, resulta muy fecundo tener en cuenta los modos de aproximación a la multitud que propone el mismo filósofo, para entender de qué manera sostienen una teoría política de la multitud.

Veámos más en detalle. Virno recupera la perspectiva de Carl Schmitt quien, en El concepto de lo político, distingue las teorías del estado y las ideas políticas a partir de la antropología que les subyace, repartiéndolas en dos campos -sobre este punto Schmitt simplifica de manera consciente-: aquellas que consideran el hombre malvado por naturaleza y aquellas que consideran el hombre bueno por naturaleza.

Sin embargo, para Schmitt (1991), las teorías políticas propiamente dichas son exclusivamente aquellas que presuponen un hombre malvado y que se estructuran a partir del eje amigo-enemigo; al contrario, las que presuponen un hombre bueno por naturaleza-Schmitt cita a las liberales y 
las anarquistas- "no desarrollan una teoría política constructiva y niegan o limitan al estado y a la esfera política que le es propia" (Marcos, 2004, p. 56).

En definitiva, como afirma Virno (2011b), desde la perspectiva del filósofo alemán, si "el Homo sapiens es un animal peligroso, inestable y (auto)destructivo, parece inevitable, para ponerle freno, la formación de un 'cuerpo político unitario' que ejerza (...) un incondicionado 'monopolio de la decisión política"” (p. 124).

En este marco, Virno encuentra una resonancia entre las palabras de Schmitt y el Freud (2001) de El malestar de la cultura, según quien la peligrosidad del homo sapiens puede ser limitada por un orden normativo, por una suerte de "compulsión de repetición que, una vez instituida, decide cuándo, dónde y cómo algo debe ser hecho, ahorrando así vacilación y dudas en todos los casos idénticos" (p. 92). Es decir que el orden normativo de las teorías políticas autoritarias ha constituido, si quisiéramos usar el vocabulario de la antropología filosófica alemana que Virno tiene muy en cuenta (en particular el trabajo de Arnold Gehlen) una suerte de corset que ha sido puesto al animal abierto al mundo y sin ambiente, con el objetivo de crearle un pseudo-ambiente.

En este sentido, la institución -entendida weberianamente como una manera de ordenar, gracias al monopolio de la fuerza, distintos tipos de acciones (Weber, 1964)- establece límites a lo que podría ser distinto de como es, en pos de hacer "seguro" el miedo.

Virno acepta las premisas político-antropológicas que han emergido hasta aquí, pero no comparte la necesidad de las conclusiones y, a la vez, recupera otra concepción de la institución. La hipótesis a la que apuesta y que llama "histórico-naturalista" 16 es planteada claramente en un fragmento que cito in extenso:

la hipótesis histórico-naturalista (...) arruinando el esquema conceptual delineado por Schmitt (...) es la siguiente: la riesgosa inestabilidad del animal humano -el llamado mal, en resumen- no implica en absoluto la formación y el mantenimiento de aquel 'supremo imperio' que es la soberanía estatal. Al contrario. El "radicalismo hostil al estado" y al modo de producción capitalista, lejos de presuponer la innata mansedumbre de nuestra especie, puede encontrar su auténtico pedestal en el pleno reconocimiento del carácter 'problemático', esto es indefinido y potencial (incluso también peligroso), del animal humano. La crítica del "monopolio de la decisión política" y en general de las instituciones cuyas reglas funcionan como una

${ }^{16}$ Sobre la concepción de historia en Virno y, más en general, en el postoperaismo, me permito remitir a mi Fagioli, $2016 \mathrm{~b}$. 
compulsión a repetir, debe apoyarse precisamente en la constatación de que el hombre es "malo por naturaleza" (Virno, 2011b, p. 126).

Entonces, para Virno el homo sapiens es un ser problemático y el lógos, lejos de constituir el espacio donde el animal con lenguaje soluciona sus conflicto, como es planteado por ejemplo por los filósofos à la Habermas ${ }^{(1989)}$, se encuentra al mismo tiempo en la base del mal y de la virtud, en la medida en que el lenguaje - por supuesto no como facultad, sino codificado en una lengua histórica o en otro sistema convencional-, puede ser usado para ordenar un bombardeo aéreo, pero también para organizar una huelga (Virno, 2005). Esta coextensividad entre peligro y reparo que reside en las condiciones bio-lingüísticas de la especie y que, como vimos, es constitutivo del modo de ser multitud, es central en la reflexión de nuestro autor.

Veamos, entonces, cuáles son los elementos principales sobre los que se apoya la teoría política de Virno. Para nuestro autor, lo que llama "paradoja de Hobbes" no tiene solución, es decir que es imposible salir del estado de naturaleza, porque este depende de aquella característica peculiar e ineliminable del homo sapiens que es la apertura al mundo y que emerge plenamente a la superficie sólo en el marco del modelo productivo postfordista.

Ahora bien. El filósofo recurre a las herramientas filosóficas que le provee Wittgenstein para fundamentar su crítica a la soberanía a través de la imposibilidad de salir del estado de naturaleza.

Si en contra del normativismo de Hans Kelsen (2015) -que termina su regreso al infinito en una norma última, la Grundnorm-, Schmitt (1994) afirma que la aplicación de la norma escapa necesariamente a la norma misma y pertenece al ámbito de la decisión, Wittgenstein le permite a Virno ir más allá de ese esquema.

En las Investigaciones filosóficas, Wittgenstein (1999) plantea que la aplicación de una norma no puede ser explicada con una norma, sino que requiere de una decisión. De hecho, como subraya Virno (2011b) en "Chiste y acción innovadora", "[d]ecidir (de caedere, 'cortar') significa, en efecto, truncar el regreso al infinito al que está condenado todo intento de fundar sobre una regla ulterior la aplicación de la regla en cuestión una y otra vez" (p. 48). De esta manera, sigue el autor, "[1]a decisión es el modo absolutamente especifico en que la praxis detiene el regreso al infinito connatural a las normas" (Virno, 2011b, p. 48). Hasta aquí tenemos un sustancial acuerdo entre su punto de vista y el de Schmitt. Sin embargo, Virno pone en tensión las perspectiva de los dos autores y escribe: "Schmitt piensa que la 'realización del derecho', es decir la decisión, es una prerrogativa 
exclusiva de la soberanía estatal. Wittgenstein piensa que la aplicación siempre problemática de la regla, es decir la decisión, es la prerrogativa de todo animal lingüístico (Virno, 2011b, p. 53). De esta manera, Virno infiere que 'cada humilde aplicación de la norma contiene siempre en sí un fragmento del estado de excepción"” (Virno, 2011b, p. 25) y concluye:

[1]a distinción no es, entonces, entre decisionismo y normativismo, sino entre dos formas disímiles de decisionismo: monopólico el primero, difusivo (mejor dicho inevitable, en tanto inscripto en la naturaleza misma del lenguaje verbal), el segundo. Y no se ha dicho en absoluto que las aplicaciones/decisiones de los hablantes no puedan volverse contra las aplicaciones/ decisiones de los soberanos (Virno, 2011b, p. 53).

Lo último que me interesa traer a colación en este apartado es la manera en que Virno piensa la institución, porque el filósofo italiano no usa esa categoría en el sentido weberiano, sino que retoma la idea de "institución pura" -la lengua- de Ferdinand de Saussure (2007) que es la matriz de todas las demás ${ }^{17}$. En este sentido, la lengua es institución pura porque tiene el efecto de estabilizar y reparar la carencia y el riesgo original del homo sapiens: la facultad de lenguaje. La lengua histórica, por lo tanto, "protege del primer y más grave peligro al que está expuesto el animal neoténico: una potencia que permanece como tal, desprovista de actos correspondientes" (Virno, 2011b, p. 153).

Para concluir este apartado. Aunque ningún parecido entre la lengua histórica en tanto institución pura y las instituciones políticas históricamente determinadas - es decir aquellas que han habitado la modernidad- es posible, Virno se hace algunas preguntas que son, desde mi punto de vista, centrales. En primer lugar su inquietud se dirige al hecho de si "es concebible una institución política, en la acepción más rigurosa de este adjetivo, que tome prestado su forma y su funcionamiento de la lengua" (Virno, 2011b, p. 156); en segunda instancia, si "es verosímil una República que proteja y estabilice al animal humano del mismo modo en que la lengua cumple su rol protector y estabilizador respecto a la facultad de lenguaje" (Virno, 2011b, p. 156); $\mathrm{y}$, finalmente, si podemos pensar en una "República insustancial basada

\footnotetext{
${ }^{17}$ La lengua es institución pura para de Saussure (2007), en el sentido de que sus signos son completamente arbitrarios, lo cual "implica teóricamente la libertad de establecer cualquier posible relación entre la materia fónica y las ideas. De aquí resulta que cada uno de esos dos elementos unidos en los signos guardan su vida propia en una proporción desconocida en otras instituciones, y que la lengua se altera, o mejor, evoluciona, bajo la influencia de todos los agentes que puedan alcanzar sea a los sonidos sea a los significados" (p. 156).
} 
en diferencias y diferencias entre diferencias, no representativa" (Virno, 2011b, p. 156).

Para estas preguntas, Virno afirma no tener respuestas, sin embargo, la cuestión del autogobierno de la multitud y su imbricación con la facultad de lenguaje, con la ambivalencia constitutiva que acarrea, es para el filósofo un problema abierto y urgente.

\section{V.}

El punto de partida de este último parágrafo es que Virno subraya la necesidad de un trabajo -teórico y militante, ça va sans dire- que siente una nueva gramática política y que permita pensar una salida del dispositivo conceptual de la modernidad. Como se puede intuir fácilmente, el autor no se refiere a una teoría política de la soberanía de signo contrario a las que critica. Si pensamos en la historia del siglo XX y en sus "alternativas", no hay ni toma del Palacio de Invierno, ni vía electoral al poder, ni democracia progresiva: el referente polémico es lisa y llanamente el poder soberano y los conceptos que necesariamente conlleva.

En Virtuosismo y revolución, un ensayo publicado en los años 90 donde retoma la distinción entre voice y exit, planteada por Albert Hirschman (1970), releída en términos de protesta y defección, el filósofo subraya la centralidad que en una teoría política de la multitud cobra una desobediencia radical.

El filósofo usa el adjetivo radical para referirse a la desobediencia civil, para indicar que esta no apela a leyes fundamentales, como por ejemplo la Constitución, para oponerse a otras leyes consideradas incoherentes o contradictorias con respecto a aquellas. Para ser radical, la desobediencia debe, en palabras de Virno (2017), "poner en cuestión la propia facultad de mandar del Estado" (p. 115). Dicho en otros términos, en vez de reivindicar las leyes fundamentales, debería cuestionar la misma condición de posibilidad de las leyes civiles, aquella obligación a obedecer que Hobbes construye teóricamente en el Tratado sobre el ciudadano (De Cive) $)^{18}$.

Según nuestro autor, la pregunta fundamental de la teoría de las instituciones, ¿por qué es necesario obedecer?, constituye un regreso ad infinitum y la manera que el filósofo inglés encuentra para sortear esa dificultad es a través del pasaje del estado de naturaleza al estado civil por medio del pacto $^{19}$. En el pacto se originan, paralelamente, tanto el monopolio

${ }^{18}$ La obligación a obedecer, sin la cual las leyes civiles no tendrían ninguna validez, precede a toda ley civil (Hobbes, 2009).

${ }^{19}$ Esto, desde el punto de vista de Virno (2011b), hace caer a Hobbes en una paradoja, en base a la cual "la obligación a la obediencia es, al mismo tiempo, causa y efecto de la 
de la decisión política -lo que Schmitt (1994) ha definido "el poder de declarar el estado de excepción"-, como el cuerpo político unitario ${ }^{20}$. Podemos concluir entonces que, para Virno, los conceptos fundamentales del pensamiento político moderno, los de soberanía y de pueblo, se fundan en una paradoja.

Ahora bien. A partir del análisis de la época histórica en cuyo marco reflexiona, el filósofo sostiene, siguiendo a Schmitt, que estamos frente al desmoronamiento - que el filósofo alemán lamenta y que Virno ve de manera favorable- del monopolio de la decisión política ${ }^{21}$. Virno (2011b) atribuye ese desmoronamiento a los cambios en el modelo productivo, al ciclo de luchas sociales de los años 60 y 70 y a "la posterior proliferación de formas de vida refractarias a un "pacto preliminar de obediencia"' (p. 132), pero lo que es más importante, desde mi punto de vista, es que para Virno esto hace concebible una esfera pública por fuera y más allá del estado. En este sentido, el verdadero desafío con el que tiene que medirse una teoría crítica a la altura de nuestros tiempos está constituido por el hecho de imaginar "cuáles son las instituciones que se colocan más allá del 'monopolio de la decisión política' encarnado en el estado" y, por otra parte, "cuáles son las instituciones a la altura del general intellect" (Colectivo Situaciones, 2011, p. 15).

Lo que llama "teoría política por venir" y en pos de la cual considera que es necesario trabajar debe ser, tal y como lo explica el autor en otro pasaje de Virtuosismo y revolución:

Un modelo de acción de pleno derecho, capaz de medirse con las "cosas últimas" de la política moderna, en fin, con los grandes temas articulados sucesivamente por Hobbes, Rousseau, Lenin, Schmitt (si se piensa en parejas fundamentales tales como mando/obediencia, público/privado, amigo/ enemigo, consenso/violencia, etc.) (Virno, 2017, p. 114).

Ahora bien. Si retomamos "El llamado 'mal' y la crítica del estado", muy importante para los temas que me interesan aquí, vemos que Virno (2011b) postula que tal vez sea posible encontrar en la narración bíblica del éxodo

existencia del estado; está sostenida por aquello de lo que constituye el fundamento" (p. 135).

${ }^{20}$ Por otra parte, para Schmitt el estado de naturaleza vuelve a aparecer en dos ocasiones, cuando el soberano efectivamente declara el estado de excepción y cuando el pueblo se descompone en multitud.

${ }^{21}$ Escribe Schmitt (1991) en el Prólogo a la edición de 1963 da Las categorías de lo político: "[1]a época de la estatalidad está llegando ya a su fin (...) El estado como modelo de la unidad política, el estado como titular del más extraordinario de todos los monopolios, esto es, del monopolio de la decisión política, está por ser destronado" (p. 90). 
"el más autorizado modelo teológico-político de ir más allá del estado"(p. 133). Las razones por las cuales el éxodo nos otorga un modelo recuperable en el marco de la contemporaneidad son dos y acarrean consecuencias que deberemos tener en cuenta. La primera es porque "señala la posibilidad de una sustracción emprendedora"(Virno, 2011b, p. 133), la segunda es porque "excluye que esta sustracción tenga como fundamento propio la natural mansedumbre del animal humano"(Virno, 2011b, p. 133).

Por lo que concierne a la primera cuestión, cabe señalar que si en este caso el éxodo al que hace referencia Virno es el éxodo del poder soberano, ese mismo problema es central en el análisis del capitalismo de nuestro autor. De hecho, su tesis es que el postfordismo nace como respuesta al éxodo de la fábrica que, junto con el rechazo al empleo estable y a determinados conocimientos del trabajo vivo, ajenos a la disciplina, moviliza la contrarrevolución capitalista. Pero ya antes, en un artículo publicado en 1981, el filósofo había individuado en la frontera abierta y en la posibilidad de éxodo, la principal dificultad encontrada por el capitalismo en su afirmación en Estados Unidos; una dificultad que se había reproducido, siglos después, con la vía de fuga representada en Europa por el welfare state. "Los niveles salariales -concluía en aquella ocasión Virno- no dependen de la plena ocupación, sino del nivel de movilidad social, de la velocidad con que los individuos transmigran hacia actividades siempre diferentes" (Virno, 1981, p. 37) ${ }^{22}$.

El éxodo, desde este punto de vista, se coloca "en las antípodas del desesperado 'lo único que podemos perder son las cadenas' [y] se enclava en una riqueza latente, en una exuberancia de posibilidades, en el principio del tertium datur" (Virno, 2003, p. 73).

Mostrando una vez más la distancia con respecto a la cultura estatista del movimiento obrero oficial, Virno opone la imaginación anticapitalista del espacio abierto, al sueño del Estado "realmente" socialista. En este sentido, el éxodo de las formas modernas de la política se presenta también como la forma lógica de una acción política basada en el general intellect. Lejos de deducir, como vimos, unas pautas de los rasgos distintivos del homo sapiens, y lejos de redactar un programa político, Virno apela a la capacidad de invención política de la multitud, una capacidad que las singularidades

${ }^{22}$ En este sentido, podríamos decir que Virno anticipa una tesis que será desarrollada a cabalidad por Yann Moulier Boutang (2006), quien ve en el control de la movilidad y en la búsqueda de garantías contra el abandono de la relación de trabajo por parte de la fuerza de trabajo, una de las necesidades básicas del capitalismo. En este mismo sentido, Sandro Mezzadra (2005) sostiene que las migraciones cobran centralidad en la historia y en el funcionamiento concreto del modo de producción capitalista. 
entrenan constantemente en el marco del capitalismo postfordistas, y que pueden usar para inventar, desde abajo, maneras de organizar la vida en común.

\section{Referencias bibliográficas}

Arendt, H. (2009). La condición humana. Paidós.

Aristóteles. (1971). Retórica. Instituto de Estudios Políticos.

Aristóteles. (2007). Ética nicomaquea. Losada.

Balestrini, N. (2006). Lo queremos todo. Traficantes de Sueños.

Berardi, F. (2009). The Soul at Work. From Alienation to Autonomy. Semiotext.

Chignola, S., \& Mezzadra, S. (2012). Fuori dalla pura politica. Laboratori globali della soggettività. Filosofia Politica, 26 (1), 65-81. http://doi.org/10.1416/36474

Colectivo Situaciones. (2011). La madurez de los tiempos: la actualidad de la multitud. Entrevista a Paolo Virno. En Virno P., Ambivalencia de la multitud. Entre la innovación y la negatividad (pp. 7-18). Tinta Limón.

de Saussure, F. (2007). Curso de lingüistica general (vol. I). Losada.

Duso, G. (2015). La representación política. Unsam Edita.

Esposito, R. (2003). Communitas. Origen y destino de la comunidad. Amorrortu.

Fagioli, A. (2016a). Política y vida. Perspectivas posoperaístas. Pléyade. Revista de humanidades y ciencias sociales, 17, 155-180.

Fagioli, A. (2016b). Antideterminismo y luchas. Hacia una concepción postoperaista del desarrollo histórico. Anacronismo e Irrupción. Revista de Teoría y Filosofía Política Clásica y Moderna, 11(6), 152-171.

Fagioli, A. (2019). Naturaleza bruta y dimensión productiva del conocimiento. La idea "materialista" de mundo en la perspectiva política de Paolo Virno. Anacronismo e Irrupción. Revista de Teoría y Filosofía Política Clásica y Moderna, 16(9), 61-77.

Fagioli, A. (2020). Biopolítica y fuerza de trabajo. Virno lee a Foucault entre Aristóteles y Marx. Revista de filosofia: Aurora, 57 (32), 790-805. http://doi. org/10.7213/1980-5934.32.057.AO02

Freud, S. (2001). Obras Completas (vol. XXI). El porvenir de una ilusión, El malestar de la cultura y otras obras (1927-1931). Amorrortu.

Fujita Hirose, J. (2006). Reading Gilbert Simondon: Transindividuality, technical activity and reification. Radical Philosophy, 136, 34-43.

Gramsci, A. (1981). Los cuadernos de la carcel (6 voll.). Era.

Habermas, J. (1989). Ciencia y Técnica como ideología. Tecnos.

Hardt, M., \& Negri, A. (2002) Imperio. Paidós.

Hardt, M., \& Negri, A. (2004) Multitud. Debate.

Hegel, G. F. W. (2006). Filosofia real. Fondo de Cultura Económica.

Heidegger, M. (2009). El ser y el tiempo. Fondo de Cultura Económica.

Hirschman, A. (1970). Exit, Voice, and Loyalty. Responses to decline in Firms, Organizations and States. Harvard University Press.

Hobbes, T. (2007). Leviatán (vol. 1). Losada. 
Hobbes, T. (2009). Tratado sobre el ciudadano. UNED.

Kelsen, H. (2015). La Teoría pura del Derecho. Eudeba.

Marazzi, Ch. (2003). El sitio de los calcetines. El giro lingüistico de la economía y sus efectos en la política. Akal.

Marcos, D. (2004). Acerca de los conceptos de política y soberanía en Carl Schmitt y Thomas Hobbes. Foro Interno, 4, 45-58.

Marx, K. (1989). Elementos fundamentales para la crítica de la economía política: borrador (vol. 2). Siglo XXI.

Marx, K. (2010). El capital. Libro primero (vol. 1). Siglo XXI.

Mazzeo, M. (2015). La nutella e gli schiaffi. Filosofia e linguaggio nell'operaismo italiano. Rivista Italiana di Filosofia del Linguaggio, 9(1), 174-192. http://doi. org/0.4396/201506ITA12

Mezzadra, S. (2005). Derecho de fuga. Migraciones, ciudadanía y globalización. Traficantes de sueños.

Milazzo, F. (2011 enero 18). Linguaggio, politica e 'natura umana'. Un'intervista a Paolo Virno. Haeccit@s. https://haecceitasweb.com/2011/01/18/linguaggiopolitica-e-natura-umana-unintervista-a-paolo-virno/.

Moulier Boutang, Y. (2006). De la esclavitud al trabajo asalariado. Economía histórica del trabajo asalariado embridado. Akal.

Negri, A. (1980). Del obrero-masa al obrero social: entrevista sobre el obrerismo. Anagrama.

Rispoli, T. (2016). Tra teoria politica e antropologia materialista. Intervista a Paolo Virno. Filosofia Italiana, 11(1). http://www.filosofiaitaliana.net/wp-content/ uploads/2018/04/Rispoli_Virno.pdf.

Schmitt, C. (1991). El concepto de lo político. Alianza.

Schmitt, C. (1994). Teología política. Struhart.

Virno, P. (1981). Marx e il sentimento dell'abbondanza. Metropoli, 6(3), 35-37.

Virno, P. (2003). Gramática de la multitud. Para un análisis de las formas de vida contemporáneas. Colihue.

Virno, P. (2005). Cuando el verbo se hace carne. Lenguaje y naturaleza humana. Traficantes de sueños.

Virno, P. (2011a). Convenzione e materialismo. L'unicità senz'aura. DeriveApprodi.

Virno, P. (2011b). Ambivalencia de la multitud. Entre la innovación y la negatividad. Tinta Limón.

Virno, P. (2017). La idea de mundo. Intelecto público y uso de la vida. La Marca.

Weber, M. (1964). Economía y sociedad. Esbozo de sociología comprensiva. Fondo de Cultura Económica.

Wittgenstein, L. (1999). Investigaciones filosófica. Altaya. 
